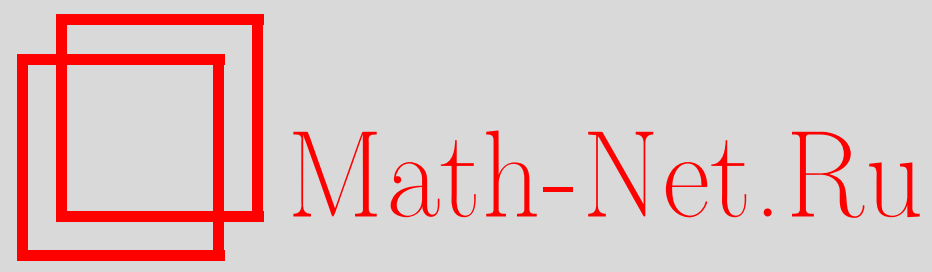

Д. С. Ананичев, М. В. Волков, Многообразия разрешимых колец Ли конечной ширины, Матем. заметки, 1998, том 63, выпуск 5, 643-650

DOI: https://doi.org/10.4213/mzm1329

Использование Общероссийского математического портала Math-Net.Ru подразумевает, что вы прочитали и согласны с пользовательским соглашением http://www.mathnet.ru/rus/agreement

Параметры загрузки:

IP: 54.92 .164 .108

26 апреля 2023 г., 04:23:55

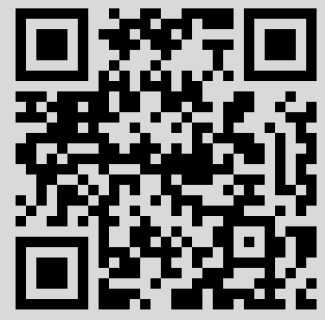




\title{
МНОГООБРАЗИЯ РАЗРЕШИМЫХ КОЛЕЦ ЛИ КОНЕЧНОЙ ШИРИНЫ
}

\author{
Д. С. Ананичев, М.В. Волков \\ Получено полное описание многообразий колец Ли, решетка подмногообразий ко- \\ торых имеет конечную шшрину. \\ Библиография: 10 названий.
}

Одним из наиболееважных объектов, ассоциированных с каждым многообразием $\mathscr{V}$, является решетка его подмногообразий $L(\mathscr{V})$. (Напомним, что операции в решетке $L(\mathscr{V})$ определяются так: объединение $\mathscr{X} \vee \mathscr{Y ~ п о д м н о г о о б р а з и и ̆ ~} \mathscr{X}$ и $\mathscr{Y ~ - ~ э т о ~ н а и м е н ь ш е е ~ м н о - ~}$ гообразие, содержащее и $\mathscr{X}$, и $\mathscr{Y}$, а пересечение $\mathscr{X} \cap \mathscr{Y ~ с о в п а д а е т ~ с ~ п е р е с е ч е н и е м ~ д а н н ы х ~}$ подмногообразий как классов.) В силу этого весьма естественно выглядит идея классификации многообразий по свойствам их решеток подмногообразий. Применительно к многообразиям колец Ли исследования в указанном направлении были инищиированы В.А. Артамоновым (см. [1], а также его обзор [2]), исследовавшим такие многообразия $\mathscr{V}$, что решетка $L(\mathscr{V})$ является цепю. В дальнейшем в литературе рассматривались многообразия колец Ли, решетки подмногообразий которых удовлетворяли таким обобщениям свойства "быть цепью”, как дистрибутивность и другие решеточные тождества (см., например, [3]). В настоящей заметке мы изучаем многообразия колец Ли с другим естественным ограничением на решетку подмногообразий, обобщающим свойство “быть цепью” в другом направлении.

Напомним, что шириной упорядоченного множества $\langle P ; \leqslant\rangle$ назьвается точная верхняя грань мощностей антицепей в $\langle P ; \leqslant\rangle$. Классическая теорема Дилуорса [4] характеризует упорядоченные множества конечной ширины $n$ как теоретико-множественные объединения в точности $n$ цепей. Будем для краткости именовать многообразия, решетки подмногообразий которых имеют конечную ширину, многообразиями конечной ширины. Целью работы является описание многообразий разрешимых лиевых колец конечной ширины.

Сформулируем основной результат работы. Для каждого простого $p$ обозначим через $\mathscr{M}_{p}$ многообразие колец Ли, задаваемое тождествами

$$
p x=0, \quad x y(z t)=0, \quad x y z^{p}=0, \quad x y^{p} x^{p-1}=0 .
$$

Здесь и ниже используется левонормированная запись, а выражение типа $y x^{n}$ служит сокращением для

$$
y \underbrace{x x \cdots x}_{n \text { раз }} .
$$

Наименьшее многообразие, содержащее данное кольцо $R$, назовем многообразием, $п$ рохсденным $R$. 
ТЕОремА. Многообразие разрешимых колеи, Ли является многообразием конечной ширины тогда и только тогда, когда оно либо порожсается некоторым конечным кольцом, либо представимо в виде обгединения многообразия $\mathscr{M}_{p}$ и некоторого многообразия, порожденного конечным кольиом.

Необходимость условия теоремы будет доказана в п. 1, достаточность - в п. 2.

1. Напомним, что многообразие, в котором каждое конечнопорожденное кольцо конечно, назьвается локально конечным. Нашей ближайшей целью будет доказательство того, что всякое многообразие разрешимых колец Ли конечной ширины локально конечно. Для этого мы воспользуемся известной характеризацией локально конечных многообразий разрешимых колец Ли на языке “запрещенных объектов". (Эта характеризация легко вытекает, например, из предложения 1 работы [5], точнее - из эквивалентности условий б) и и) этого предложения). Пусть $p$ - простое число, $A_{p}$ - абелева алгебра Ли над полем $\mathrm{GF}(p)$ с базисом $a_{0}, a_{1}, \ldots, a_{n}, \ldots, s$ - дифференцирование алгебры $A_{p}$, определенное правилом $a_{n} s=a_{n+1}$ для всех $n=0,1, \ldots$. Через $S_{p}$ мы обозначим полупрямое произведение $A_{p} \lambda \mathrm{GF}(p) s$, а через $\mathscr{A}_{0}$ - многообразие всех абелевых колец Ли. Многообразие, порожденное кольцом $R$, будем обозначать через $\operatorname{var} R$.

Лемма 1. Многообразие разрешимых колеи Ли локально конечно тогда и только тогда, когда оно не содержит ни одного из многообразий $\mathscr{A}_{0} u \operatorname{var} S_{p}$.

В силу леммы 1 , чтобы доказать, что всякое многообразие разрешимых колец Ли конечной ширины локально конечно, достаточно убедиться, что ни одно из многообразий $\mathscr{A}_{0}$ и $\operatorname{var} S_{p}$ не является многообразием конечной ширины. Это очевидно для многообразия $\mathscr{A}_{0}$, ибо хорошо известно, что решетка $L\left(\mathscr{A}_{0}\right)$ изоморфна решетке неотрицательных целых чисел по делимости и, стало быть, содержит бесконечную антицеп. Зафиксируем теперь простое число $p$ и займемся вычислением ширины решетки $L\left(\operatorname{var} S_{p}\right)$. Заметим, что эта решетка обстоятельно изучалась в $[3, \S 2]$, где было показано, например, что среди ее факторов встречаются конечные простые решетки сколь угодно большой длины. Можно проверить, однако, что все эти простые решетки имеют ширину всего лишь 4 и, таким образом, построения из [3] не позволяют ответить на интересуюший нас сейчас вопрос.

Для произвольного натурального $k$ обозначим через $I_{k}$ идеал кольца $S_{p}$, порожденный элементом $a_{0}-a_{k(p-1)}$, а через $T_{k}$ - соответствуюшее факторкольцо $S_{p} / I_{k}$.

Лемма 2. Кольцо $T_{k}$ удовлетворяет тождеству $х у z=x y z^{1+k(p-1)}$.

ДоКАЗАТЕЛЬСтво. Достаточно показать, что для любых элементов $x, y, z \in S_{p}$ разность $x y z-x y z^{1+k(p-1)}$ лежит в идеале $I_{k}$. Ясно, что произведение $x y$ принадлежит коммутанту $A_{p}$ кольца $S_{p}$. Представим его как линейную комбинацию элементов базиса пространства $A_{p}$ :

$$
x y=\alpha_{1} a_{i_{1}}+\alpha_{2} a_{i_{2}}+\cdots+\alpha_{m} a_{i_{m}}=\alpha_{1} a_{0} s^{i_{1}}+\alpha_{2} a_{0} s^{i_{2}}+\cdots+\alpha_{m} a_{0} s^{i_{m}},
$$

где $\alpha_{1}, \alpha_{2}, \ldots, \alpha_{m} \in \mathrm{GF}(p)$. Запишем еше элемент $z$ в виде суммы $z=a+\lambda s$, где $a \in A_{p}$, $\lambda \in \operatorname{GF}(p)$. Тогда из $(2)$ получаем

$$
\begin{aligned}
x y z & =\lambda\left(\alpha_{1} a_{0} s^{i_{1}+1}+\alpha_{2} a_{0} s^{i_{2}+1}+\cdots+\alpha_{m} a_{0} s^{i_{m}+1}\right), \\
x y z^{1+k(p-1)} & =\lambda^{1+k(p-1)}\left(\alpha_{1} a_{k(p-1)} s^{i_{1}+1}+\alpha_{2} a_{k(p-1)} s^{i_{2}+1}+\cdots+\alpha_{m} a_{k(p-1)} s^{i_{m}+1}\right) .
\end{aligned}
$$


Вычитая из первого равенства второе и учитывая, что $\lambda^{1+k(p-1)}=\lambda$ для любого $\lambda \in$ $\mathrm{GF}(p)$, приходим к равенству

$$
\begin{aligned}
x y z-x y z^{1+k(p-1)}= & \lambda\left(\alpha_{1}\left(a_{0}-a_{k(p-1)}\right) s^{i_{1}+1}+\alpha_{2}\left(a_{0}-a_{k(p-1)}\right) s^{i_{2}+1}+\cdots\right. \\
& \left.+\alpha_{m}\left(a_{0}-a_{k(p-1)}\right) s^{i_{m}+1}\right)
\end{aligned}
$$

которое и показьвает, что элемент $x y z-x y z^{1+k(p-1)}$ принадлежит идеалу $I_{k}$.

Лемма 3. Если $k$ не делит $n$, то кольцо $T_{k}$ не удовлетворяет тождеству $x y z=x y z^{1+n(p-1)}$.

ДокаЗАтЕльство. Достаточно найти такие $x, y, z \in S_{p}$, что $x y z-x y z^{1+n(p-1)} \notin I_{k}$. Пусть $x=a_{0}, y=s, z=s$. Тогда $x y z=a_{2}$, а $x y z^{1+n(p-1)}=a_{2+n(p-1)}$. Покажем, что если $k$ не делит $n$, то $a_{2}-a_{2+n(p-1)}$ не принадлежит идеалу $I_{k}$. Предположим противное. Тогда для некоторых коэффициентов $\alpha_{1}, \ldots, \alpha_{m} \in \mathrm{GF}(p)$ и неотрицательных целых показателей $i_{1}, \ldots, i_{m}$ вьполнено равенство

$a_{2}-a_{2+n(p-1)}=\alpha_{1}\left(a_{0}-a_{k(p-1)}\right) s^{i_{1}}+\alpha_{2}\left(a_{0}-a_{k(p-1)}\right) s^{i_{2}}+\cdots+\alpha_{m}\left(a_{0}-a_{k(p-1)}\right) s^{i_{m}}$.

Представим правую часть (3) в виде

$$
\alpha_{1}\left(a_{i_{1}}-a_{i_{1}+k(p-1)}\right)+\alpha_{2}\left(a_{i_{2}}-a_{i_{2}+k(p-1)}\right)+\cdots+\alpha_{m}\left(a_{i_{m}}-a_{i_{m}+k(p-1)}\right)
$$

и подсчитаем для этого выражения сумму всех коэффициентов при таких базисных элементах $a_{l}$, что индекс $l$ дает остаток 2 при делении на $k(p-1)$. Ясно, что эта сумма равна 0, но для левой части (3) при условии, что $k$ не делит $n$, соответствующая сумма равна 1. Таким образом, равенство (3) невозможно.

Из лемм 2 и 3 немедленно вытекает, что все многообразия вида $\operatorname{var} T_{q}$, где $q-$ простое число, попарно несравнимы. Таким образом, мы получаем

СледствИЕ. Решетка $L\left(\operatorname{var} S_{p}\right)$ имеет бесконечную ширину.

Как уже обсуждалось, вместе с леммой 1 это дает

ПРЕДЛОЖЕНИЕ. Многообразие разрешимых колеи, Ли конечной ширины локально конечно.

Многообразие называется кроссовым, если оно локально конечно, конечно базируемо и содержит лишь конечное число подмногообразий. (Такое определение кроссовости равносильно стандартному, см., например, $[6, \S 1]$.) В силу основного результата работы Бахтурина и Ольшанского [7] (см. такжеглаву 7 монографии [8]) многообразиеколец Ли будет кроссовым тогда и только тогда, когда оно порождается некоторым конечным кольцом.

Почти кроссовым назьвается некроссово многообразие, все собственные подмногообразия которого кроссовы. Легко понять, что каждое некроссово многообразие содержит некоторое почти кроссово подмногообразие. Разрешимые почти кроссовы многообразия колец Ли описаны Ю.А. Бахтуриньм и А.Ю. Ольшанским в [9] (см. также $[8, \S 7.8])$ - это многообразие $\mathscr{A}_{0}$ всех абелевых колец Ли и упоминавшаяся во введении серия многообразий $\mathscr{M}_{p}(p$ - простое число), задаваемых тождествами $(1)$. 
Приступим непосредственно к доказательству необходимости. Пусть $\mathscr{V}$ - произвольное многообразие разрешимых колец Ли конечной ширины. Если $\mathscr{V}$ кроссово, доказывать нечего. Поэтому считаем, что $\mathscr{V}$ некроссово, а тогда $\mathscr{V}$ должно содержать некоторое почти кроссово многообразие. Поскольку, как уже отмечалось, $\mathscr{A}_{0}$ не является многообразием конечной ширины, $\mathscr{V}$ содержит многообразие $\mathscr{M}_{p}$ для некоторого простого $p$. Если $\mathscr{V}=\mathscr{M}_{p}$, то снова доказывать нечего. Если же $\mathscr{V} \supset \mathscr{M}_{p}$, то поскольку $\mathscr{V}$ - локально конечное многообразие, а каждое локально конечное многообразие порождается своими конечными кольцами, найдется конечное кольцо $K_{1} \in \mathscr{V} \backslash \mathscr{M}_{p}$. Положим $\mathscr{K}_{1}=\operatorname{var} K_{1}$ и $\mathscr{V}_{1}=\mathscr{M}_{p} \vee \mathscr{K}_{1}$. Если $\mathscr{V}=\mathscr{V}_{1}$, то все доказано. В противном случае найдется конечное кольцо $K_{2} \in \mathscr{V} \backslash \mathscr{V}_{1}$, и можно рассмотреть многообразие $\mathscr{K}_{2}=\operatorname{var}\left(K_{1} \times K_{2}\right)$ и многообразие $\mathscr{V}_{2}=\mathscr{M}_{p} \vee \mathscr{K}_{2}$. Продолжая описанный процесс, мы либо остановимся на некотором шаге, получив, что $\mathscr{V}=\mathscr{M}_{p} \vee \mathscr{K}_{n}$ для некоторого многообразия $\mathscr{K}_{n}$, порожденного конечным кольцом, и тем самьм докажем необходимость условия теоремы, либо построим бесконечную строго возрастающую последовательность

$$
\mathscr{K}_{1} \subset \mathscr{K}_{2} \subset \cdots \subset \mathscr{K}_{n} \subset \cdots
$$

кроссовых подмногообразий многообразия $\mathscr{V}$. Докажем, что вторая возможность на самом деле не реализуется.

Отметим, что по построению ни одно из многообразий $\mathscr{K}_{n}$ не содержится в $\mathscr{M}_{p}$ и

$$
\mathscr{M}_{p} \vee \mathscr{K}_{n} \subset \mathscr{M}_{p} \vee \mathscr{K}_{m}
$$

при $n<m$. Для каждого натурального $c$ обозначим через $\mathscr{M}_{p}^{c}$ подмногообразие многообразия $\mathscr{M}_{p}$, заданное внутри $\mathscr{M}_{p}$ тождеством

$$
x_{1} \cdots x_{c}=0
$$

Для натурального $c$, кратного $p$, обозначим через $\mathscr{N}_{p}^{c}$ подмногообразие многообразия $\mathscr{M}_{p}$, заданное внутри $\mathscr{M}_{p}$ тождеством

$$
\sum_{i=2}^{c} x_{1} x_{i} x_{2} \cdots x_{i-1} x_{i+1} \cdots x_{c}=0 .
$$

В.А. Артамоновым $[1$, теорема 3$]$ показано, что решетка $L\left(\mathscr{M}_{p}\right)$ является цепью типа $\omega+1$ и имеет следуюший вид:

$$
\begin{gathered}
\mathscr{M}_{p}^{1} \subset \mathscr{M}_{p}^{2} \subset \cdots \subset \mathscr{M}_{p}^{p} \subseteq \mathscr{N}_{p}^{p} \subset \mathscr{M}_{p}^{p+1} \subset \mathscr{M}_{p}^{p+2} \subset \cdots \\
\subset \mathscr{M}_{p}^{2 p} \subset \mathscr{N}_{p}^{2 p} \subset \mathscr{M}_{p}^{2 p+1} \subset \mathscr{M}_{p}^{2 p+2} \subset \cdots \subset \mathscr{M}_{p}
\end{gathered}
$$

где нестрогое включение $\mathscr{M}_{p}^{p} \subseteq \mathcal{N}_{p}^{p}$ обрашается в равенство только при $p=2$. Пусть $w$ - ширина решетки $L(\mathscr{V})$. Зафиксируем $k>w$ и подберем индекс $c$ так, чтобы многообразия $\mathscr{M}_{p}^{c+1}$ и $\mathscr{K}_{k}$ были несравнимы. Это возможно, поскольку многообразие $\mathscr{K}_{k}$ не содержится в $\mathscr{M}_{p}^{c}$ ни для какого $c$, а если бы для всех $c$ многообразия $\mathscr{M}_{p}^{c}$ содержались бы в $\mathscr{K}_{k}$, то решетка $L\left(\mathscr{K}_{k}\right)$ была бы бесконечной в противоречие с кроссовостью $\mathscr{K}_{k}$. Ясно, что если $\mathscr{K}_{k}$ несравнимо с $\mathscr{M}_{p}^{c+1}$, то для всех $i, j=1, \ldots, k$ многообразия $\mathscr{K}_{j}$ 


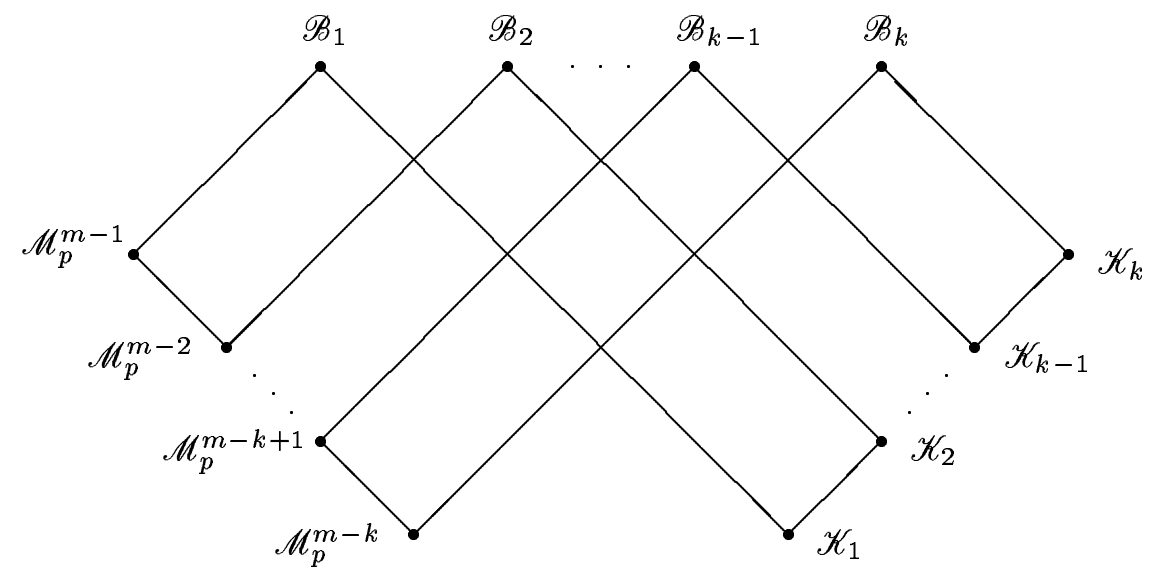

Рис. 1

и $\mathscr{M}_{p}^{c+i}$ несравнимы. Положим $m=c+k+1$ и для каждого $i=1, \ldots, k$ рассмотрим объединение $\mathscr{B}_{i}=\mathscr{M}_{p}^{m-i} \vee \mathscr{K}_{i}$. Наши построения иллюстрирует рис. 1.

Так как ширина решетки $L(\mathscr{V})$ меньше $k$, найдутся такие индексы $i$ и $j$, что $i<j$, но многообразия $\mathscr{B}_{i}$ и $\mathscr{B}_{j}$ сравнимы. Предположим сначала, что $\mathscr{B}_{i} \subseteq \mathscr{B}_{j}$. Тогда в силу модулярности решетки многообразий и включения $\mathscr{M}_{p}^{m-i} \supset \mathscr{M}_{p}^{m-j}$ получаем

$\mathscr{M}_{p}^{m-i}=\mathscr{M}_{p}^{m-i} \cap \mathscr{B}_{i} \subseteq \mathscr{M}_{p}^{m-i} \cap \mathscr{B}_{j}=\mathscr{M}_{p}^{m-i} \cap\left(\mathscr{M}_{p}^{m-j} \vee \mathscr{K}_{j}\right)=\mathscr{M}_{p}^{m-j} \vee\left(\mathscr{M}_{p}^{m-i} \cap \mathscr{K}_{j}\right)$

Поскольку $L\left(\mathscr{M}_{p}\right)$ - цепь и $\mathscr{M}_{p}^{m-i} \supset \mathscr{M}_{p}^{m-j}$, из (6) следует, что $\mathscr{M}_{p}^{m-i}=\mathscr{M}_{p}^{m-i} \cap \mathscr{K}_{j}$. Это противоречит несравнимости многообразий $\mathscr{M}_{p}^{m-i}$ и $\mathscr{K}_{j}$.

Рассмотрим теперь случай, когда $\mathscr{B}_{j} \subseteq \mathscr{B}_{i}$. В силу модулярности и включения $\mathscr{K}_{i} \subset \mathscr{K}_{j}$ имеем

$$
\mathscr{K}_{j}=\mathscr{K}_{j} \cap \mathscr{B}_{j} \subseteq \mathscr{K}_{j} \cap \mathscr{B}_{i}=\mathscr{K}_{j} \cap\left(\mathscr{M}_{p}^{m-i} \vee \mathscr{K}_{i}\right)=\mathscr{K}_{i} \vee\left(\mathscr{M}_{p}^{m-i} \cap \mathscr{K}_{j}\right) .
$$

Из (7) вытекает, что $\mathscr{K}_{j} \subseteq \mathscr{K}_{i} \vee \mathscr{M}_{p}$, а следовательно, и $\mathscr{K}_{j} \vee \mathscr{M}_{p} \subseteq \mathscr{K}_{i} \vee \mathscr{M}_{p}$. Это снова приводит к противоречию, ибо по построению последовательности $\left\{\mathscr{K}_{n}\right\}$ для всех $i<j$ выполняется строгое включение $\mathscr{M}_{p} \vee \mathscr{K}_{i} \subset \mathscr{M}_{p} \vee \mathscr{K}_{j}$.

Итак, описанньй вьше процесс должен оборваться, и необходимость условия теоремы доказана.

2. Выше уже отмечалось, что многообразие, порожденное конечным кольцом Ли, кроссово, в частности, содержит лишь конечное число подмногообразий и потому является многообразием конечной ширины. Остается проверить, что многообразием конечной ширины будет и объединение произвольного многообразия $\mathscr{K}$, порожденного конечным кольцом Ли, с любым многообразием вида $\mathscr{M}_{p}$.

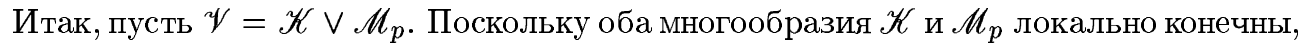
локально конечным, очевидно, будет и их объединение $\mathscr{V}$. Нам потребуется одно полезное свойство энгелевых колец локально конечных разрешимых многообразий, подмеченное в [5] (см. там предложения 1 и 3 ). Напомним, что элемент $e$ решетки $L$ называется нейтральным, если для любых $a, b \in L$ подрешетка, порожденная $a, b$ и $e$, дистрибутивна. 
Лемма 4. В любом локально конечном многообразии И разрешимых колеи, Ли совокупность всех әнгелевых колец, образует подмногообразие, являющееся нейтральным әлементом решетки $L(\mathscr{U})$.

Пусть $\mathscr{E}$ - многообразие, состоящее из всех энгелевых колец нашего многообразия $\mathscr{V}$. Следующая лемма сводит стоящую перед нами задачу к доказательству конечности ширины многообразия $\mathscr{E}$.

ЛЕмМА 5. Если $\mathscr{E}$ - многообразие конечной иирины, то таково и многообразие $\mathcal{V}$.

ДоКАЗАТЕльСтво. Прежде всего, заметим, что многообразие $\mathscr{M}_{p}$ энгелево (этовидно из тождества $x y z^{p}=0$ в (1)). Поэтому $\mathscr{M}_{p} \subseteq \mathscr{E}$. Отсюда следует, что $\mathscr{V}=\mathscr{E} \vee \mathscr{K}$.

Если $\mathscr{X}$ и $\mathscr{Y}$ - подмногообразия в $\mathscr{V}$ такие, что $\mathscr{X} \subseteq \mathscr{Y}$, будем обозначать через $[\mathscr{X}, \mathscr{Y}]$ интервал решетки $L(\mathscr{V})$ с концами $\mathscr{X}$ и $\mathscr{Y}$. По известному свойству модулярных решеток интервалы $[\mathscr{E}, \mathscr{V}]=[\mathscr{E}, \mathscr{E} \vee \mathscr{K}]$ и $[\mathscr{E} \cap \mathscr{K}, \mathscr{K}]$ изоморфны. Так как решетка $L(\mathscr{K})$ конечна, отсюда, в частности, вытекает, что интервал $[\mathscr{E}, \mathscr{V}]$ конечен. Пусть $m$ - число элементов в этом интервале, а $w$ - ширина решетки $L(\mathscr{E})$. Покажем, что ширина решетки $L(\mathscr{V})$ не превосходит $m w$.

В самом деле, пусть $\mathscr{X}_{1}, \ldots, \mathscr{X}_{m w+1}$ - произвольные подмногообразия в $\mathscr{V}$. Среди объединений $\mathscr{X}_{1} \vee \mathscr{E}, \ldots, \mathscr{X}_{m w+1} \vee \mathscr{E}$ не более $m$ различных; поэтому, перенумеровав многообразия, можно считать, что $\mathscr{X}_{1} \vee \mathscr{E}=\mathscr{X}_{2} \vee \mathscr{E}=\cdots=\mathscr{X}_{w+1} \vee \mathscr{E}$. Далее, среди пересечений $\mathscr{X}_{1} \cap \mathscr{E}, \ldots, \mathscr{X}_{w+1} \cap \mathscr{E}$ не более $w$ несравнимых; поэтому $\mathscr{X}_{i} \cap \mathscr{E} \subseteq \mathscr{X}_{j} \cap \mathscr{E}$ для некоторых различных $i, j \in\{1, \ldots, w+1\}$. В силу леммы 4 многообразия $\mathscr{X}_{i}, \mathscr{X}_{j}$ и $\mathscr{E}$ порождают дистрибутивную подрешетку решетки $L(\mathscr{V})$. Вычисляя в этой подрешетке, получаем

$$
\mathscr{X}_{i}=\mathscr{X}_{i} \cap\left(\mathscr{X}_{i} \vee \mathscr{E}\right)=\mathscr{X}_{i} \cap\left(\mathscr{X}_{j} \vee \mathscr{E}\right)=\left(\mathscr{X}_{i} \cap \mathscr{X}_{j}\right) \vee\left(\mathscr{X}_{i} \cap \mathscr{E}\right) \subseteq\left(\mathscr{X}_{i} \cap \mathscr{X}_{j}\right) \vee\left(\mathscr{X}_{j} \cap \mathscr{E}\right) \subseteq \mathscr{X}_{j}
$$

Таким образом, среди произвольных $m w+1$ элементов решетки $L(\mathscr{V})$ нашлась пара сравнимых, что и означает, что ширина $L(\mathscr{V})$ не превосходит $m w$.

В соответствии с доказанной редукцией займемся теперь оценкой ширины решетки $L(\mathscr{E})$. Заметим прежде всего, что

$$
\mathscr{E}=\mathscr{E} \cap \mathscr{V}=\mathscr{E} \cap\left(\mathscr{K} \vee \mathscr{M}_{p}\right)=(\mathscr{E} \cap \mathscr{K}) \vee \mathscr{M}_{p}
$$

(мы воспользовались здесь включением $\mathscr{M}_{p} \subseteq \mathscr{E}$ и модулярностью решетки многообразий). Подмногообразие кроссова многообразия само кроссово, поэтому пересечение $\mathscr{E} \cap \mathscr{K}$ также порождается конечным кольцом, причем это кольцо должно быть энгелевым. Конечное энгелево кольцо нильпотентно, стало быть, $\mathscr{E} \cap \mathscr{K}$ - нильпотентное многообразие. Обозначим через $с$ его индекс нильпотентности, т.е. наименьшее число с тем свойством, что в $\mathscr{E} \cap \mathscr{K}$ вьполнено тождество $x_{1} \cdots x_{c}=0$, а через $\mathscr{E}_{c}$ - многообразие всех нильпотентных индекса $\leqslant c$ колециз $\mathscr{E}$. Тогда $\mathscr{E} \cap \mathscr{K} \subseteq \mathscr{E}_{c}$ и в силу (8) мы имеем $\mathscr{E}=\mathscr{E}_{c} \vee \mathscr{M}_{p}$. Отметим еще, что $\mathscr{E}_{c}-$ кроссово многообразие (как локально конечное нильпотентное многообразие).

Нам потребуется еще одно свойство многообразия $\mathscr{M}_{p}$, легко извлекаемое из результатов работы [1] (см. там доказательство теоремы 3). 
ЛЕмма 6. Произвольное полилинейное тождество $f\left(x_{1}, \ldots, x_{d}\right)=0$, не выполняюиееся в $\mathscr{M}_{p}$, влечет внутри $\mathscr{M}_{p}$ тохдество $x_{1} \cdots x_{d+1}=0$.

Лемма 7. Пусть $\mathscr{X}$ - произвольное подмногообразие в $\mathscr{E}$, не содержащееся в $\mathscr{E}_{c}$. Если пересечение $\mathscr{X} \cap \mathscr{M}_{p}$ удовлетворяет некоторому полилинейному тожсдетву $f\left(x_{1}, \ldots, x_{d}\right)=0$, не выполняюшемуся в $\mathscr{M}_{p}$, то әтому тожидеству удовлетворяет и все многообразие $\mathscr{X}$.

ДокАЗАТЕЛЬСТво. В условиях леммы многообразие $\mathscr{X}$ должно удовлетворять некоторому тождеству вида

$$
f\left(x_{1}, \ldots, x_{d}\right)=g\left(x_{1}, \ldots, x_{d}\right),
$$

где $g\left(x_{1}, \ldots, x_{d}\right)=0$ - тождество многообразия $\mathscr{M}_{p}$. Можно считать, что многочлен $g\left(x_{1}, \ldots, x_{d}\right)$ в $(9)$ действительно зависит от всех фигурирующих в его записи переменных, а тогда его нижняя степень больше либо равна $d$. Допустим, что $d \geqslant c$. Так как $\mathscr{E}=\mathscr{E}_{c} \vee \mathscr{M}_{p}$, все тождества многообразия $\mathscr{M}_{p}$ с нижней степенью, большей либо равной $c$, выполняются и в многообразии $\mathscr{E}$, а следовательно, и в $\mathscr{X}$. Поэтому $g\left(x_{1}, \ldots\right.$, $\left.x_{d}\right)=0$ в $\mathscr{X}$, и в силу $(9) f\left(x_{1}, \ldots, x_{d}\right)=0$ в $\mathscr{X}$. Теперь допустим, что, напротив, $d<c$. Тогда в силу леммы 6 тождество $f\left(x_{1}, \ldots, x_{d}\right)=0$ влечет внутри $\mathscr{M}_{p}$ тождество $x_{1} \cdots x_{c}=0$. Отсюда пересечение $\mathscr{X} \cap \mathscr{M}_{p}$ удовлетворяет тождеству $x_{1} \cdots x_{c}=0$, и по уже доказанному тождество $x_{1} \cdots x_{c}=0$ будет вьполнено во всем многообразии $\mathscr{X}$. Но тогда $\mathscr{X} \subseteq \mathscr{E}_{c}$, что невозможно.

Теперь мы готовы к доказательству решающей леммы.

ЛЕМма 8. Пусть $\mathscr{X ~ и ~ У ~ - ~ п р о и з в о л ь н ы е ~ п о д м н о г о о б р а з и я ~ в ~} \mathscr{E}$, не содержащиеся в $\mathscr{E}_{c}$. Toгдa

$$
\mathscr{M}_{p} \cap(\mathscr{X} \vee \mathscr{Y})=\left(\mathscr{M}_{p} \cap \mathscr{X}\right) \vee\left(\mathscr{M}_{p} \cap \mathscr{Y}\right)
$$

ДокАЗАтЕльство. Допустим, что равенство (10) нарушается для каких-то многообразий $\mathscr{X}$ и $\mathscr{Y}$. Тогда его левая часть строго содержит правую. Из описания решетки $L\left(\mathscr{M}_{p}\right)$ (см. доказательство необходимости вьше) вытекает, что найдется полилинейное тождество $f\left(x_{1}, \ldots, x_{d}\right)=0$ (в действительности, тождество одного из видов (4) или $(5))$, вьполненное в $\left(\mathscr{M}_{p} \cap \mathscr{X}\right) \vee\left(\mathscr{M}_{p} \cap \mathscr{Y}\right)$ и ложное в $\mathscr{M}_{p} \cap(\mathscr{X} \vee \mathscr{Y})$. Однако, применяя лемму 7 , мы можем заключить, что тождество $f\left(x_{1}, \ldots, x_{d}\right)=0$ вьполняется в каждом из многообразий $\mathscr{X}$ и $\mathscr{Y}$, а стало быть, и в их объединении $\mathscr{X} \vee \mathscr{Y}$. Отсюда $f\left(x_{1}, \ldots, x_{d}\right)=0$ заведомо вьполняется в $\mathscr{M}_{p} \cap(\mathscr{X} \vee \mathscr{Y})$ - противоречие.

Завершим доказательство достаточности, для чего в силу леммы 5 остается проверить, что $\mathscr{E}$ - многообразие конечной ширины. Напомним, что многообразие $\mathscr{E}_{c}$ всех нильпотентных индекса $\leqslant c$ колец из $\mathscr{E}$ кроссово, в частности, содержит лишь конечное число подмногообразий. Обозначим это число через $m$ и покажем, что ширина решетки $L(\mathscr{E})$ не превосходит $2 m$. Пусть $\mathscr{X}_{1}, \ldots, \mathscr{X}_{2 m+1}-$ произвольные подмногообразия в $\mathscr{E}$. Из них не более $m$ содержатся в $\mathscr{E}_{c}$. Следовательно, мы можем считать, что многообразия $\mathscr{X}_{1}, \ldots, \mathscr{X}_{m+1}$ удовлетворяют посылке леммы 8. Далее, интервалы $\left[\mathscr{M}_{p}, \mathscr{E}\right]=\left[\mathscr{M}_{p}, \mathscr{E}_{c} \vee \mathscr{M}_{p}\right]$ и $\left[\mathscr{M}_{p} \cap \mathscr{E}_{c}, \mathscr{E}_{c}\right]$ изоморфны, поэтому между $\mathscr{M}_{p}$ и $\mathscr{E}$ заключено не более $m$ многообразий. Следовательно, среди объединений $\mathscr{X}_{1} \vee \mathscr{M}_{p}, \ldots, \mathscr{X}_{m+1} \vee \mathscr{M}_{p}$ не более $m$ различных, и найдутся такие различные индексы $i$ и $j$, что

$$
\mathscr{X}_{i} \vee \mathscr{M}_{p}=\mathscr{X}_{j} \vee \mathscr{M}_{p}
$$


Поскольку решетка $L\left(\mathscr{M}_{p}\right)$ - цепь, можно считать, что

$$
\mathscr{X}_{i} \cap \mathscr{M}_{p} \subseteq \mathscr{X}_{j} \cap \mathscr{M}_{p}
$$

Известно [10, теорема II.12], что если три элемента произвольной модулярной решетки $L$ связаны каким-либо соотношением дистрибутивности, то они порождают дистрибутивную подрешетку в $L$. В силу этого факта и леммы 8 многообразия $\mathscr{X}_{i}, \mathscr{X}_{j}$ и $\mathscr{M}_{p}$ порождают дистрибутивную подрешетку в $L(\mathscr{E})$. Вычисляя в этой подрешетке, получаем

$$
\begin{aligned}
\mathscr{X}_{i} & =\mathscr{X}_{i} \cap\left(\mathscr{X}_{i} \vee \mathscr{M}_{p}\right)=\mathscr{X}_{i} \cap\left(\mathscr{X}_{j} \vee \mathscr{M}_{p}\right)=\left(\mathscr{X}_{i} \cap \mathscr{X}_{j}\right) \vee\left(\mathscr{X}_{i} \cap \mathscr{M}_{p}\right) \\
& \subseteq\left(\mathscr{X}_{i} \cap \mathscr{X}_{j}\right) \vee\left(\mathscr{X}_{j} \cap \mathscr{M}_{p}\right) \subseteq \mathscr{X}_{j} .
\end{aligned}
$$

Итак, $\mathscr{X}_{i} \subseteq \mathscr{X}_{j}$, т.е. среди произвольных $2 m+1$ элементов решетки $L(\mathscr{E})$ нашлось два сравнимых. Достаточность условия теоремы доказана.

\section{СПИСОК ЦИТИРОВАННОЙ ЛИТЕРАТУРЫ}

[1] Артамонов В.А. Цепные многообразия линейных алгебр // Тр. ММО. 1973. Т. 29. C. $51-78$.

[2] Артамонов В.А. Решетки многообразий линейных алгебр // УМН. 1978. Т. 33. № 2. C. $135-167$.

[3] Ананичев Д. С. Почти дистрибутивные многообразия колец Ли // Матем. сб. 1995. Т. 186. № 4. C. 3-20.

[4] Dilworth R.P. A decomposition theorem for partially ordered sets // Ann. of Math. 1950. V. 51. №1. P. 161-166.

[5] Волков М. В., Гейн А. Г. Тождества почти нильпотентных колец Ли // Матем. сб. 1982. Т. 118. №1. С. 132-142.

[6] Львов И. В. О многообразиях ассоциативных колец. I // Алгебра и логика. 1973. Т. 12. № 3. С. 269-297.

[7] Бахтурин Ю. А., Ольшанский А. Ю. Тождественные соотношения в конечных кольцах Ли // Матем. сб. 1975. Т. 96. № 4.. С. 543-559.

[8] Бахтурин Ю. А. Тождества в алгебрах Ли. М.: Наука, 1985.

[9] Бахтурин Ю. А., Ольшанский А. Ю. Разрешимые почти кроссовы многообразия колец Ли // Матем. сб. 1976. Т. 100. №3. С. 384-399.

[10] Биркгоф Г. Теория решеток. М.: Наука, 1984.

Уральский государственный университет, г. Екатеринбург

Поступило

E-mail: Dmitry.Ananichev@usu.ru, Mikhail.Volkov@usu.ru

18.11 .96 